\title{
SUSTITUCIÓN DE LA HARINA DE PESCADO POR ENSILADO BIOLÓGICO DE PESCADO EN RACIONES PARA JUVENILES DE GAMITANA, Colossoma macropomum
}

\author{
Palmira Padilla P. ${ }^{1}$, Fernando Alcántara B. ${ }^{1}$ y Juan García T. ${ }^{1}$
}

\section{RESUMEN}

Juveniles de gamitana, Colossoma macropomum, fueron alimentados con raciones peletizadas, constituidas por una ración patrón y tres niveles de sustitución de harina de pescado por ensilado biológico de pescado.

El experimento se realizó en las instalaciones del Centro Regional de Investigaciones de Loreto, del Instituto de Investigaciones de la Amazonía Peruana, en 20 jaulas de madera y malla sintética, con una capacidad de 578 litros cada una, colocadas en un estanque de tierra de $2440 \mathrm{~m}^{2}$.

En cada una de las jaulas se colocó 15 peces con una longitud y peso promedio de 22 cm y 198 g, respectivamente, efectuándose la adaptación de los peces a las condiciones experimentales, durante cinco días.

El experimento se efectuó con un diseño completamente al azar, con cuatro tratamientos y cinco repeticiones, durante 120 días, en los que se suministró alimento a los peces, dos veces al día, a razón de $3 \%$ de la biomasa.

El análisis de varianza (ANOVA), mostró que no existe diferencia significativa en el crecimiento y producción de gamitana, en cultivo con ensilado biológico de pescado y harina de pescado $(\mathrm{P}>0,05)$.

Las condiciones físico químicas del agua del estanque fueron monitoreadas periódicamente, observándose variaciones dentro de límites normales.

Palabras clave : Ensilado biológico de pescado, raciones para gamitana, Colossoma macropomum.

1 Instituto de Investigaciones de la Amazonía Peruana - IIAP. Programa de Ecosistemas Acuáticos. Centro Regional de Investigaciones de Loreto. Apartado 784. Fax 265527. Telef. 265515/ 265516 


\begin{abstract}
Results of a feeding experiment with young gamitana, Colossoma macropomum, are presented. Fishes were fed on four different pelletized diet, one standard diet and the others containing three different levels of fish silage replacing fish meal as main protein resource.
\end{abstract}

The experiments were carried out at Centro Regional de Investigaciones de Loreto Instituto de Investigaciones de la Amazonía Peruana-IIAP. Iquitos. Perú, in 20 cages, 578 1. capacity each, raised in a $2440 \mathrm{~m}^{2}$ earth pond.

Group of 15 fishes with initial means of 22,38 cm length and 197,78 g. were used in this experiments.

During the 120 days experiments, the fishes were fed twice a day, at a rate of $3 \%$ of the biomass, seven days a week.

A five days period was allowed for the fish to adapt themselves to experimental conditions. After this time, the first sample was codified and fish were measured and weighed. This procedure was repeated five times with 30 days interval.

The physical and chemical conditions of the water in the pond were checked throughout the experiment by measuring the daily water temperature and the dissolved oxygen, and $\mathrm{pH}$ once a week.

The statical analysis of fish growth indicated that there was no significant difference $(p>0,005)$ between the fish fed with fish silage and that fed on the standard diet containing fish meal.

\title{
1. INTRODUCCIÓN
}

La piscicultura en la Amazonía está en proceso de crecimiento, a pesar de su dependencia de la escasa disponibilidad de ingredientes regionales para la formulación de raciones (Saint-Paul \& Werder, 1977). Esta situación es aún más crítica, si se tiene en cuenta la dependencia de la harina de pescado como fuente de proteína, en los climas tropicales, como ha sido reportado por Hepher et al.(1978).

Sin embargo, se ha señalado también que existen en la región muchas fuentes de materia prima para la alimentación de animales, incluyendo los peces, las aves y sus 
subproductos, frutos y semillas de áreas inundables (Roubach, 1991), y macrófitas acuáticas. Pero estas posibilidades han sido poco exploradas.

De otro lado, el desarrollo y rentabilidad de los cultivos dependen, inevitablemente, de la obtención de dietas que satisfagan los requerimientos nutricionales de las especies, a fin de asegurar su crecimiento óptimo (Cantelmo, 1989).

La gamitana, Colossoma macropomum, es una especie básicamente omnívora y con gran potencial para la piscicultura (Goulding \& Carvalho, 1982; Saldaña \& Lopez, 1988), que se cultiva utilizando raciones con harina de pescado como fuente de proteína. Sin embargo, este ingrediente puede sustituirse con ensilado biológico de pescado, debido a la considerable calidad y alta digestibilidad de este último producto (Lupin, 1983; Bertullo, 1992).

El objetivo de este trabajo fue determinar los niveles de sustitución de la harina de pescado por ensilado biológico de pescado, que permitan el más alto rendimiento al más bajo costo.

\section{MATERIAL Y MÉTODOS}

El fermento biológico se preparó de acuerdo a las recomendaciones de FAO (1985), en base a los siguientes ingredientes: repollo -Brassica sp.- (41)\%, harina de trigo (17)\%, sal de cocina (3)\%, papaya -Carica papaya- (31)\% y vinagre (8)\%.

Se utilizaron peces forrajeros de las especies bujurqui, Cichlassoma bimaculatum, y mojara, Gymnocorymbus thayeri, molidos en fresco, como materia prima para la preparación del ensilado. La masa resultante se mezcló con los demás ingredientes, en las proporciones siguientes: Harina de trigo 30\% peso del pescado (p/p), sal de cocina $4 \% \mathrm{p} / \mathrm{p}$ y fermento biológico $10 \% \mathrm{p} / \mathrm{p}$. La mezcla fue homogeneizada y acondicionada en bandeja de plástico cubierta con lámina del mismo material, a temperatura ambiental $\left(30 \pm 2{ }^{\circ} \mathrm{C}\right)$ durante un período de seis días, para crear condiciones anaeróbicas. Cada 24 horas se realizó la homogeneización de la mezcla con una espátula de madera, tomándose una muestra para determinar el $\mathrm{pH}$ y el índice de acidez en ácido láctico, de acuerdo a las normas analíticas del Instituto Adolfo Lutz (1985).

Luego de seis días de hidrólisis se evaluó las características organolépticas y se expuso la mezcla al sol sobre una lámina plástica, por un período de 48 horas, al 
cabo del cual se evaluó el rendimiento del ensilado semi seco, según la ecuación de Villela de Andrade et al. (1989):

$$
\mathrm{R} \%=\mathrm{Pf} / \mathrm{Pi}
$$

donde:

$$
\begin{array}{lll}
\mathrm{R} \% & = & \text { rendimiento expresado en porcentaje } \\
\mathrm{Pf} & = & \text { peso final } \\
\mathrm{Pi} & = & \text { peso inicial }
\end{array}
$$

Se formuló cuatro raciones, en las cuales los ingredientes: harina de maíz, polvillo de arroz, harina de soya, harina de trigo y premezcla de vitaminas y minerales, se mantuvieron constantes. Las fuentes proteicas (harina de pescado y ensilado biológico de pescado), variaron en proporción inversa de 0 a $29 \%$ del total de cada ración. Todas las raciones fueron preparadas en forma de peletz secos con $5 \mathrm{~mm}$ de diámetro y almacenadas en bolsas plásticas a temperatura ambiental $\left(30 \pm 3{ }^{\circ} \mathrm{C}\right)$. Tabla 1.

Tabla 1. Composición porcentual de las raciones experimentales.

\begin{tabular}{|l|c|c|c|c|}
\hline \multirow{2}{*}{\multicolumn{1}{|c|}{ INGREDIENTES }} & \multicolumn{4}{c|}{ RACIONES } \\
\cline { 2 - 5 } & $\mathbf{R 1}$ & $\mathbf{R 2}$ & $\mathbf{R 3}$ & $\mathbf{R 4}$ \\
\hline Harina de pescado & 29,00 & 19,30 & 9,70 & 00,00 \\
\hline Ensilado & 00,00 & 9,70 & 19,30 & 29,00 \\
\hline Harina de maíz & 25,00 & 25,00 & 25,00 & 25,00 \\
\hline Polvillo de arroz & 30,00 & 30,00 & 30,00 & 30,00 \\
\hline Harina de soya & 10,00 & 10,00 & 10,00 & 10,00 \\
\hline Harina de trigo & 5,00 & 5,00 & 5,00 & 5,00 \\
\hline Minevit & 1,00 & 1,00 & 1,00 & 1,00 \\
\hline
\end{tabular}

Antes de la formulación de las raciones se efectuó el análisis de composición bromatológica, tanto de la harina de pescado, como del ensilado biológico de pescado, según la A.O.A.C. (1975) y del sistema de ingredientes de alimentos de América Latina (Weende). Tabla 2. 
El cálculo de la energía bruta (EB) se efectuó de acuerdo a White et al. (1964), Halver (1972) y Jaramillo (1988), utilizando los siguientes factores de conversión: $5,65 \mathrm{kcal} / \mathrm{g}, 4,15 \mathrm{kcal} / \mathrm{g}$ y $9,40 \mathrm{kcal} / \mathrm{g}$ para proteínas, carbohidratos y lípidos, respectivamente.

Los juveniles de gamitana, Colossoma macropomum, utilizados en el experimento, se obtuvieron por reproducción artificial en el Centro Regional de Investigaciones de Loreto. La longitud y peso promedio iniciales de los especímenes fue de $22 \mathrm{~cm}$ y $198 \mathrm{~g}$, respectivamente.

Se utilizó 20 jaulas de $0,85 \times 0,85 \times 1,00 \mathrm{~m}$. construidas con listones de madera de $1 \times 2$ pulgadas y malla plástica de $2 \mathrm{~mm}$ de diámetro, con una capacidad individual de $578 \mathrm{l}$. que fueron colocadas dejando $20 \mathrm{~cm}$ fuera del agua, en un estanque de tierra de $2440 \mathrm{~m}^{2}$. La tasa de siembra fue de 15 peces por jaula.

Antes de la siembra se sometió a los peces a adaptación a las condiciones experimentales por un período de cinco días, al cabo del cual se determinó la longitud y el peso inicial promedios.

Diariamente se proporcionó alimento a los peces a razón del 3\% de su biomasa en cada jaula. La ración calculada se ofreció dos veces al día, a las 10.00 y 14.00 horas.

La conversión alimenticia (CAA) se calculó según la fórmula de Rangel (1987), a través de la relación de la ganancia en peso promedio final y el peso inicial de cada tratamiento y la ración consumida aparente $(\mathrm{RC})$, durante el experimento, según la ecuación:

$$
\mathrm{CAA}=\mathrm{GP}=\frac{\text { Peso promedio final }- \text { Peso promedio inicial }}{\text { Cantidad de ración proporcionada }}
$$

Mensualmente se efectuó muestreos de crecimiento al 100\% de peces, suspendiendo la alimentación un día antes de cada muestreo.

Las evaluaciones de crecimiento y de variación de la composición corporal de los peces se efectuaron siguiendo un lineamiento completamente al azar, según Banzatto \& Kronka (1989).

La temperatura del agua del estanque de cultivo se evaluó dos veces al día, durante la mañana y la tarde, semanalmente se evaluó el oxígeno disuelto y el $\mathrm{pH}$ del agua. 


\section{RESULTADOS Y DISCUSIÓN}

En la figura 1 se observa las variaciones del pH y la acidez del ensilado después de seis días de incubación a temperatura ambiental.

El fermento biológico permitió hacer variaciones de $\mathrm{pH}$ y acidez de la mezcla de pescado molido, asegurando su preservación, como ya fue reportado por Lupin (1983) y Adams et al. (1987), debido a que las bacterias productoras de ácido láctico utilizan la harina de trigo como materia prima para la fermentación (Areche et al., 1989).

Las variaciones de $\mathrm{pH}$ de 4,5 a 4,0 y acidez en ácido láctico de 2,8 a 3,5 \%, observadas en el ensilado, son similares a las obtenidas por Lupin (1983), Adams et al. (1987), Villela de Andrade (1989), Arthur (1991), Ximenes-Carneiro (1991), Areche et al. (1992), Lessi et al. (1992) y Padilla (1995) en ensilado de residuo de pescado.

Las características organolépticas del ensilado observadas a los seis días coinciden con las reportadas por Bertullo (1992) y están dadas por: color castaño oscuro, textura cremosa, sabor agridulce levemente amargo, olor ácido suave; características que son propias del ensilado biológico que tiene como fuente de carbohidratos a la harina de trigo y que corresponden a la categoría de buena calidad, señalada por Ximenes-Carneiro (1991), Bertullo (1992), Lessi et al. (1992) y Padilla (1995).

El ensilado expuesto al sol durante 48 horas a temperatura ambiental de $35 \pm 3{ }^{\circ} \mathrm{C}$ alcanzó un tenor de humedad de 22,20\% y un rendimiento de $50 \%$ del total, resultado que es aproximado al obtenido por Villela de Andrade (1989), que con un tenor de humedad de $10 \%$, obtuvo un rendimiento de $46,8 \%$, evitando de esta manera el crecimiento de hongos. Resultados similares obtuvieron Ximenes-Carneiro (1991) y Padilla (1985), que alcanzaron rendimientos de 51\% y 55,2\%. 
Tabla 2. Composición bromatológica del ensilado biológico de pescado y de la harina de pescado.

\begin{tabular}{|l|c|c|}
\hline \multicolumn{1}{|c|}{ COMPOSICIÓN } & HARINA DE PESCADO & ENSILADO BIOLÓGICO \\
\hline Lípidos & 3,40 & 9,20 \\
Proteína & 56,70 & 30,20 \\
Humedad & 20,60 & 22,20 \\
Fibra bruta & 0,00 & 0,40 \\
Ceniza & 18,90 & 18,20 \\
Carbohidratos & 0,00 & 19,80 \\
Energía kcal/100 MS & 260,18 & 252,85 \\
\hline
\end{tabular}

Las raciones formuladas presentaron características diferenciales, tales como: La ración R1 presentó mayor tenor de proteína bruta y ceniza y, a la vez, menor tenor de extracto no nitrogenado y extracto etéreo y, por consiguiente, menor energía bruta; la ración $\mathrm{R} 4$ presentó menor tenor de proteína bruta, ceniza y fibra bruta y, a la vez, mayor tenor de extracto no nitrogenado, lípidos y energía bruta; las raciones R2 y R3 presentaron niveles de proteína bruta intermedios (24 y 22 , respectivamente).

A su vez, las cuatro dietas presentaron tenores de lípidos variados, con niveles entre 7,82 y 9,88 , por debajo del nivel de $10 \%$ recomendado por Carpenter en Ottati \& Bello (1992) y coincidiendo con Villela de Andrade (1989), que reporta que los niveles altos pueden causar problemas de aceptabilidad de las raciones que contienen ensilado o harina de pescado, por el aparecimiento de sabor de pescado en la carne de animales alimentados con tales raciones. Tabla 3.

Tabla 3. Composición bramatológica de las raciones experimentales.

\begin{tabular}{|c|c|c|c|c|c|c|}
\hline Raciones & PB & E E & EN N & M M & F B & E B \\
\hline R1 & 25,90 & 7,82 & 37,55 & 13,78 & 4,86 & 375,66 \\
\hline R2 & 24,00 & 8,50 & 40,51 & 10,47 & 4,82 & 383,61 \\
\hline R3 & 22,12 & 9,19 & 43,41 & 10,46 & 4,79 & 391,48 \\
\hline R4 & 20,22 & 9,88 & 46,35 & 9,00 & 4,75 & 399,45 \\
\hline
\end{tabular}

$\mathrm{PB}=$ Proteína bruta $; \mathrm{EE}=$ Extracto etéreo $; \mathrm{ENN}=$ Extracto no nitrogenado; $\mathrm{MM}=$ Material mineral; $\mathrm{FB}=$ Fibra bruta; $\mathrm{EB}=$ Energía bruta $(\mathrm{kcal} / 100 \mathrm{~g}$ de materia seca $)$ 
El mayor incremento de peso se observó en el tratamiento con la ración R2, en que se sustituyó el 9,7\% de la harina de pescado, por ensilado biológico de pescado, alcanzándose un peso final de $570 \pm 28 \mathrm{~g}(\mathrm{cv}=12,3 \%)$. El incremento de peso de la ración $\mathrm{R} 1$, en la que no se substituyó la harina de pescado y R4 en la que la substitución fue completa, fue menor que en la ración $\mathrm{R} 2$, alcanzándose un peso final de $516 \pm 18 \mathrm{~g}(\mathrm{cv}=77 \%)$ y $472 \pm 24 \mathrm{~g}(\mathrm{cv}=11 \%)$, respectivamente. De otro lado, en la ración R3 en que se substituyó el 19,3\% de la harina de pescado, se observó el incremento más bajo alcanzándose un peso final de 466 $20 \mathrm{~g}(\mathrm{cv}=4 \%)$. Figura 2.

La ganancia de peso por día de los peces (Tabla 4), según las raciones administradas, presentó la siguiente distribución: R1 $(2,65)>$ R2 $(2,58)>$ R4 $(2,29)>$ R3 $(2,24)$.

No se observó diferencias significativas en el peso de los peces al finalizar el experimento $(\mathrm{p}>0,05)$

La sustitución de ingredientes normalmente incluidos en raciones, por otras fuentes alternativas, ocurre con frecuencia (Campos y Padilla, 1986; Ximenes-Carneiro, 1991; Padilla, 1995). Sin embargo, la mayor parte de los estudios de nutrición de peces tropicales están orientados hacia la calidad de los alimentos y muy poco a los requerimientos nutricionales (Saint-Paul \& Werder, 1977). Al respecto, Saint-Paul (1986), trabajando con dos raciones para gamitana, Colossoma macropomum, con un tenor de proteína bruta de 27,5 y 42,1\%, durante 68 días, obtuvo una ganancia de peso de 0,8 y 0,9 g/día, respectivamente. Asimismo, Ximenes-Carneiro (1991) y Padilla (1995), trabajando con la misma especie, obtuvieron una ganancia de peso de 0,53 y $0,52 \mathrm{~g} /$ día, respectivamente. Estos valores de ganancia de peso por día son muy inferiores a los obtenidos en este trabajo. Tabla 4.

La conversión alimenticia varió de 3,1 a 3,6, niveles que se consideran satisfactorios, donde el tratamiento $\mathrm{R} 1$ presentó la menor tasa de conversión, con valores muy próximos en los tratamientos R2, R3 y R4, estadísticamente no significativos ( $\mathrm{P}>0,05)$. Tabla 5.

Existen controversias entre autores sobre la eficiencia alimenticia en razón a que cada cual utiliza diferentes ingredientes en una ración. Según Bertullo, en apuntes de Avdalov et al. (1984), la digestibilidad del ensilado es cerca de $100 \%$ y la de la harina de pescado, fluctúa entre 75 y 80\%. Asimismo, Hardy et al. (1984), manifiestaron que el uso de ingredientes que contienen altos niveles de aminoácidos libres y pequeños péptidos, pueden causar reducción en la disponibilidad biológica de la lisina y de otros aminoácidos. 
Los valores encontrados en este trabajo son similares a los obtenidos por Roubach (1991); Ximenes-Carneiro (1991) y Padilla (1995), que utilizaron diferentes fuentes de proteína.

Durante la ejecución del experimento se observó una tasa de sobrevivencia del 100\%, no obstante el manipuleo a que fueron sometidas las gamitanas, en forma mensual, con ocasión de los muestreos; lo que corrobora la resistencia de la especie y su aptitud para este tipo de cultivos.

Las condiciones físico químicas del estanque permanecieron dentro de límites normales, observándose una temperatura mínima de 28,5 y una máxima de $30{ }^{\circ} \mathrm{C}$ y un nivel de oxígeno de 2,5 y pH de 6,5, similares a los reportados por Ximenes-Carneiro (1991) y Padilla (1995). Los valores mínimos de oxígeno disuelto de 2,5 mg/litro dieron las condiciones ambientales adecuadas para el cultivo de la gamitana, concordando con VAL (1986). Al respecto, cabe señalar que, según Braun \& Junk (1982) y Saint-Paul (1986), la gamitana es un pez que puede tolerar bajas concentraciones de oxígeno disuelto y puede sobrevivir en aguas con tenores de $0,5 \mathrm{mg} / \mathrm{l}$.

Tabla 4. Promedio mensual de peso (g) de los peces, según tratamiento.

\begin{tabular}{|c|c|c|c|c|c|c|}
\hline $\begin{array}{c}\text { Trata- } \\
\text { miento }\end{array}$ & $\begin{array}{c}\text { Primer } \\
\text { mes }\end{array}$ & $\begin{array}{c}\text { Segundo } \\
\text { mes }\end{array}$ & $\begin{array}{c}\text { Tercer } \\
\text { mes }\end{array}$ & $\begin{array}{c}\text { Cuarto } \\
\text { mes }\end{array}$ & $\begin{array}{c}\text { Quinto } \\
\text { mes }\end{array}$ & $\begin{array}{c}\text { Gananc. } \\
\text { de peso } \\
\text { g/día }\end{array}$ \\
\hline $\mathrm{R} 1$ & $\begin{array}{c}198 \pm 4,8 \\
\mathrm{cv}=5,4 \%\end{array}$ & $\begin{array}{c}258 \pm 8,9 \\
\mathrm{cv}=7,7 \%\end{array}$ & $\begin{array}{c}337 \pm 14 \\
\mathrm{cv}=9,4 \%\end{array}$ & $\begin{array}{c}399 \pm 26 \\
\mathrm{cv}=15 \%\end{array}$ & $\begin{array}{c}517 \pm 18 \\
\mathrm{cv}=7,7 \%\end{array}$ & 2,65 \\
\hline $\mathrm{R} 2$ & $198 \pm 2,1$ & $255 \pm 3,6$ & $346 \pm 10$ & $430 \pm 13$ & $570 \pm 28$ & 2,58 \\
$\mathrm{cv}=2,3 \%$ & $\mathrm{cv}=3,2 \%$ & $\mathrm{cv}=6,7 \%$ & $\mathrm{cv}=65,0 \%$ & $\mathrm{cv}=12,3$ & \\
\hline $\mathrm{R} 3$ & $198 \pm 4,6$ & $249 \pm 6,5$ & $356 \pm 12$ \\
$\mathrm{cv}=5,2 \%$ & $\mathrm{cv}=5,9 \%$ & $\mathrm{cv}=7,9$ & $\begin{array}{c}367 \pm 10 \\
\mathrm{cv}=6,3 \%\end{array}$ & $\begin{array}{c}466 \pm 20 \\
\mathrm{cv}=9,4 \%\end{array}$ & 2,24 \\
\hline $\mathrm{R} 4$ & $198 \pm 4,9$ & $256 \pm 4,3$ & $335 \pm 5,6$ & $391 \pm 16$ & $\begin{array}{c}373 \pm 24 \\
\mathrm{cv}=11 \%\end{array}$ & 2,29 \\
& $\mathrm{cv}=5,5 \%$ & $\mathrm{cv}=3,8 \%$ & $\mathrm{cv}=3,7 \%$ & $\mathrm{cv}=8,8 \%$ & $\mathrm{cv}=11 \%$ & \\
\hline
\end{tabular}


Tabla 5. Conversión alimenticia de los peces.

\begin{tabular}{|l|c|c|c|c|}
\hline Ración & Pf $(g)$ & Pi $(g)$ & GP $(g)$ & C A A \\
\hline R1 & 38,750 & 14,823 & 23,927 & 3.1 \\
\hline R2 & 38,050 & 14,833 & 23,217 & 3.3 \\
\hline R3 & 34,985 & 14,838 & 20,147 & 3.6 \\
\hline R4 & 35,450 & 14,841 & 20,609 & 3.6 \\
\hline
\end{tabular}

$\mathrm{CAA}=$ conversión alimenticia aparente. $\mathrm{Pf}=$ peso final.

$\mathrm{Pi}=$ peso inicial. $\mathrm{GP}=$ ganancia de peso.

El costo de las raciones utilizadas en el experimento varió entre 1,70 y 1,30 soles por kilogramo, para las raciones R1 y R4, respectivamente; correspondiendo un valor de 1,60 soles por kilogramo a la ración $\mathrm{R} 2$, que presentó el mayor rendimiento.

El análisis costo beneficio del cultivo demuestra que con la dieta R2 se obtienen los más altos beneficios por la venta del pescado. Tablas 6,7 y 8 .

Tabla 6. Costos según tipo de dieta (Soles = 2,65 US \$) .

\begin{tabular}{|l|r|r|r|r|}
\hline Item & \multicolumn{1}{|c|}{$\mathrm{R} 1$} & $\mathrm{R} 2$ & \multicolumn{1}{c|}{$\mathrm{R} 3$} & \multicolumn{1}{c|}{$\mathrm{R} 4$} \\
\hline Alevinos & 20,00 & 20,00 & 20,00 & 20,00 \\
Jaulas & 33,00 & 33,00 & 33,00 & 33,00 \\
Alimento & 127,84 & 123,43 & 105,66 & 99,01 \\
Pago obrero & 62,50 & 62,50 & 62,50 & 62,50 \\
\hline Tatal & 243,34 & 238,93 & 221,16 & 214,51 \\
\hline
\end{tabular}

Tabla 7. Ingresos según tipo de dieta (Soles = 2,65 us \$).

\begin{tabular}{|c|c|c|c|}
\hline R1 & R2 & R3 & R4 \\
\hline 310,00 & 342,20 & 280,00 & 283,60 \\
\hline
\end{tabular}

Precio/kg de gamitana en mercado 8,00 soles.

Tabla 8. Beneficio según tipo de dieta (Soles).

\begin{tabular}{|c|c|c|c|}
\hline R1 & R2 & R3 & R4 \\
\hline 66,66 & 103,27 & 58,84 & 69,09 \\
\hline
\end{tabular}


Figura 1. Variación del pH y del ácido láctico del ensilado biológico

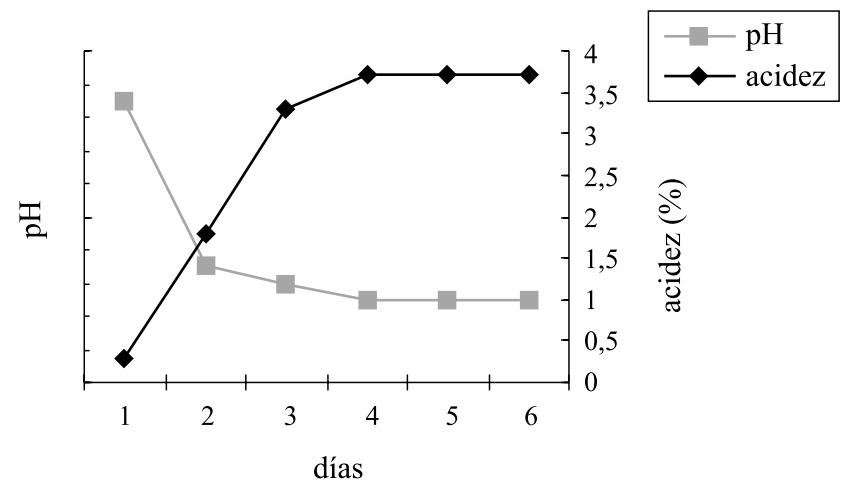

Figura 2. Crecimiento en peso de los peces

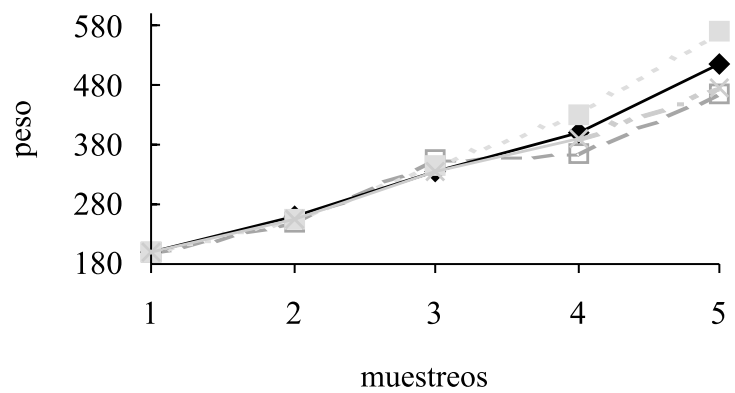

$\longrightarrow-\mathrm{R} 1 \cdots \mathrm{R} 2-\square-\mathrm{R} 3 \stackrel{\mathrm{x}}{-}-\mathrm{R} 4$ 


\section{BIBLIOGRAFÍA}

ADAMS, M.R.; COOKE, R.D.; TWIDDY,D.R. 1987. Fermentation Parameters involved in the production of lactic acid preserved fish-glucose substrates. Int. J. Fd. Sci. Tecnol. Vol. (22): 105-114.

ARECHE, N.T.; BERENZ,Z.V.; LEON,G.O.1989. Desarrollo de ensilado de residuo de pescado utilizando bacterias lácticas del yogur. In: Consulta de expertos sobre tecnología de productos pesqueros en América Latina 2. Montevideo. Roma, FAO. 14p.

ARTHUR, L.M.S.R. Utilizaçao de ensilado biológico de peixe na eleboraçao de uma racao para desenvolvimento de pós-larvas de caramarao de água doce Macrobrachium rosenbergiim M. Dissertacao de Mestrado, URFFJ. Rio de Janeiro 138 p.

ASSOCIATION OF OFFICIAL ANALYTICAL CHEMISTS-A.O.A.C. 1975. Official Methods of Analysis. 12a. Edition. George Banta Co. INC, Manasha, Wisconsin, 937 pp.

AVDALOV, N.; BARLOCOO, N.; BAUZA, R.; BERTULLO, E.T.; CORENGIA, C.; GIACOMETTI, L.; PANUCIO, A. 1989. Evaluación del ensilaje biológico de pescado en la alimentación de cerdos en engorde. In: Consulta de Expertos sobre tecnología de Productos pesqueros en América Latina 2. Montevideo. Roma. FAO, 20 p.

BANZATTO, D.A. \& KRONKA, S. DO N. 1989. Experimentaçao agrícola. Departamento de Ciëncias Exatas. Facultade de Ciencias Agrárias e Veterinárias - UNESP. Jaboticabal. SP. 247 pp.

BELLO,R.A.; GUTIÉRREZ,M.; OTTATI,M.; MARTINES,A.1992. Estudios sobre la elaboración de ensilado de pescado por vía microbiana en Venezuela. $2^{a}$ Consulta de Expertos sobre Tecnología de productos Pesqueros en América Latina. Montevideo, Uruguay, 11-15 de Diciembre de 1989. Informe de pesca 441. Supl. Roma. FAO. 369p.

BERTULLO, E. 1992. Ensilado de pescado en la pesquería artesanal. $2^{a}$ Consulta de Expertos sobre Tecnología de productos Pesqueros en América Latina. Montevideo, Uruguay, 11-15 de Diciembre de 1989. Informe de pesca 441. Supl. Roma. FAO. 368p. 
BRAUN, E. JUNK, W.J. 1982. Morfological adaptation of two amazonian caracoids (Pisces for surviving oxigen deficent waters). Intertional Rivue der Gesamten Hidrobiologie. Vol. 67. n 6:869-886.

CAMPOS, B.L. \& PADILLA, P.P. 1986. Efectos del "kudzú" (Pueraria phaseoloides, Leguminosae) y del "cetico" (Cecropia sp., Moraceae), como fuentes proteicas en la alimentación de la "gamitana", Colossoma macropomum, Cuvier 1818. Boletín Técnico n 1. Instituto de Investigación de la Amazonía Peruana. IIAP - Iquitos- Perú 12 pp.

CANTELMO, O. \& SOUZA, J.A. 1987. Influencia da alimentação de niveis protéicos para o desenvolvimento inicial do pacú Colossoma mitrei. In: Sintese de trabalhos realizados com espécies do genero Colossoma. Projeto Aquicultura. CPTS. Pirassununga,SP

CANTELMO, O.A. 1989. Nutrição de peixes e Aquicultura. In: Cultivo de Colossoma. Hernández, R. A. (edit). Bogotá. Red Regional de Entidades y Centros de Acuicultura de América Latina. Primera Reunión de grupos de Trabajo Técnico, Pirassununga. SUDEPE. CIID, COLCIENCIAS. 84-91p.

DISNEY, J.G. \& JAMES, D. 1979. Fish silage production and its use. FAO. Fish. Rep., 230: Roma 105p.

FAO, 1985. Relatório de tecnología e Controle de Qualidade de productos de pesca. Praia, Rep. de Cabo Verde, 27/11 a 11/12 de 1984. Roma. 24p.

FAO, 1989. El estado mundial de la agricultura y la alimentación. Colección FAO Agricultura 21. Roma, 163p.

HALVER, J.E. 1972. Fish Nutition. Academic Press. New York. And London. 713 pp.

HARDY, R.W.; SHEARER, K.D.; SPINELLI, J. 1984. The nutitional properties of co-dried fish silage in rainbow trout, Salmno gairdneri, dry diets. Aquaculture, 38:35-44.

HASSAN, T.E. \& HEATH, J.L. 1986. Biological fermentation of fish waste for potencial use in animal and poultry. Agricultural Waste, 15: 1-15.

HEPHER, B.; SANDBANK, E.; SHELEF, G. 1978. Alternative protein sources for warm water fish. EIFAC/78/SYMPS:11.2. Hamburg ii +29 p pre prints. 
INSTITUTO ADOLFO LUTZ. 1985. Normas Analíticas. Vol. I, Métodos Químicos e Físicos para Análise de alimentos. Säo Paulo. 371p.

JARAMILLO, N.D. 1988. Alimentación de peces. Requerimientos, Cálculos de raciones. Materias primas y dietas. Universidad de Caldas. Facultad de Medicina Veterinaria y Zootécnica. Centro de Investigaciones Piscícolas. Manizales. Colombia. 35pp.

LESSI, E., XIMENES-CARNEIRO, A.R.; LUPIN, H.M. 1992. Obtenção de ensilado biológico de peixe. In: $2^{a}$ Consulta de Expertos sobre Tecnología de productos Pesqueros en América Latina. Montevideo, Uruguay, 11-15 de Diciembre de 1989. Informe de pesca 441. Supl. Roma. FAO. 368p.

LINDGREN, S. \& PLEJE, M. 1983. Silage fermentation of fish on fish waste products with lactic acid bacteria. J. Sci. Fd. Agric. 34: 1057-1067.

LUPIN, H.M. 1989. Ensilado biológico de pescado. Una propuesta para la utilización de residuos de la pesca continental en América Latina. FAO. COPESCAL/83/10.

KOMPIANG, I.P. \& CRESWEEL, D.C. 1979. Microbial fish silage: chemical composition, fermentation characteristics and nutritional value. In: Fish Silage Production and Its Use, DISNEY, J.G. \& JAMES, D. (Eds). FAO.

MACKIE, I.M.; HARDY,R.; HOBBS, G. 1971. Poisson fermenté et produits derivés. RAPPORTES DE LA FAO SUR LESPECHES. FIIP/100. Roma. 62p.

OTTATI, G.M. \& BELLO, R.A. 1992. Ensilado microbiano de pescado en la alimentación porcina II. Valor nutritivo de los productos en dietas para cerdos. 2da. Consulta de Expertos sobre Tecnología de Productos Pesqueros en América Latina. Montevideo, Uruguay, 11-15 de Diciembre de 1989. Informe de pesca 441. Supl. Roma. FAO. 368p.

PADILLA, P.P.P. 1995. Influência do Ensilado Biológico de Peixe e do Peixe cozido no crescimento e composiçao corporal de Alevinos de Tambaqui, Colossoma macropumum, (Cuvier, 1818). Dissertação de Mestrado. Instituto Nacional de Pesquisas da Amazónia/ Fundação Universidade de Amazonas. Manaus. 76p. 
RANGEL, M.F.S. 1987. Nutrição de peixes. In: Manual de Pesca. Associação dos Engenheiros de Pesca do Estado do Ceará. Fortaleza-CE. Ogawa \& Kroeke. (edits). P. $257-277$.

RODRÍGUEZ, V.G.; FEDOR, A.B.; CONTRERAS, P.R.; FLORES, G.R.; NAVARRO, G.G.; EZQUERRA, M.A.; PÉREZ, C.L. 1989. Definición tecnológica para la elaboración de hidrolizado de proteína a partir de la fauna acompañante del camarón de la plataforma cubana. In: Consulta de Expertos sobre tecnología de productos Pesqueros América Latina 2. Montevideo. Roma, FAO. 12p.

ROUBACH, R. 1991. Uso de frutos e sementes de florestas inundáveis na alimentação de tambaqui, Colossoma macropomum, (Cuvier 1818). Dissertação de Mestrado. INPA-FUA 79pp.

SAINT-PAUL, U. \& WERDER, U. 1977. Aspectos generales sobre la piscicultura en Amazonas y resultados preliminares de experimentos de alimentación con raciones peletizadas con diferentes composiciones. Simp. Asoc. Lat. Acui. I. Maracay. Venezuela. 22 pp.

SAINT-PAUL, U. 1986. Potential for aquaculture of south American fresh water fishes: a review. Aquaculture, 54: 205-240.

SALDAÑA, A.L. LÓPEZ, M.M.P.1988. Formulación y evaluación de dietas para Colossoma macropomum en Méjico. An. VI Symp. Lat. E V Symp. Bras. de Aquic. Florianópolis. SC. 323-344.

VAL, A.L. 1986. Hemoglobinas de Colossoma macropomum. Aspectos adaptativos. (Ilha de Marchanteria, Manaus, AM). Tese de Doutorado. INPA/FUA. 112p.

VAN WYK, G.N. \& HEYDENRICH, C.M.S. 1985. The production of naturally fermented fish silage using varius Lactobacilli and different carbohydrate sources. J. Sci. Fd. Agric., 36: 1093-1103.

VILLELA DE ANDRADE, M.F.; LESSI, E.; FRANQUEIRA DA SILVA, J.M. 1989. Obtención de ensilado de residuo de sardina, Sardinella brasiliensis, y su empleo en la formulación de raciones de mínimo costo para aves. In: Consulta de Expertos sobre tecnología de Productos Pesqueros en América Latina 2. Montevideo. Roma. FAO. 19p. 
XIMENES-CARNEIRO, A.R. 1991. Elaboração e uso de ensilado biológico de pescado na alimentação de alevinos de tambaqui, Colossoma macropomum, (Cuvier, 1818). Dissertação de Mestrado. Instituto Nacional de Pesquisas da Amazónia/Fundaçao Universidade de Amazonas. Manaus. 81p. 